\title{
Discrimination and decoherence of Schrödinger cat states in lossy quantum channels
}

\author{
R. K. Goncharov ${ }^{1,2}$, A. D. Kiselev ${ }^{1,2}$, N. G. Veselkova ${ }^{1}$, Ranim Ali $^{3}$, F. D. Kiselev ${ }^{1,2}$ \\ ${ }^{1}$ Quantum Information Laboratory, ITMO University, Kadetskaya Line, 3, \\ 199034 Saint Petersburg, Russia \\ ${ }^{2}$ Laboratory of Quantum Processes and Measurements, ITMO University, Kadetskaya Line, 3, \\ 199034 Saint Petersburg, Russia \\ ${ }^{3}$ Faculty of Photonics, ITMO University, Kronverksky Pr. 49, bldg. A, \\ 197101 Saint Petersburg, Russia \\ rkgoncharov@itmo.ru
}

\begin{abstract}
We investigate environment induced effects of decoherence in discrimination between the Schrödinger cat states transmitted through noisy quantum channels such as optical fibers. We calculate the fidelity and the statistics of photocounts for both even and odd coherent states. The method that uses the beam splitter-like transformation acting in the enlarged Hilbert space to model the quantum channel is compared with the approach based on the Lindblad dynamics of one-mode bosonic systems.
\end{abstract}

Keywords: Schrödinger cat states, decoherence, quantum state discrimination.

Received: 20 November 2021

Revised: 30 November 2021

\section{Introduction}

Quantum states known as the even (odd) Schrödinger cat states, $\left|\alpha_{+}\right\rangle\left(\left|\alpha_{-}\right\rangle\right)$, are represented by symmetric (antisymmetric) superpositions of the coherent states:

$$
\left|\alpha_{ \pm}\right\rangle=\frac{1}{\sqrt{M_{ \pm}(\alpha)}}(|\alpha\rangle \pm|-\alpha\rangle)
$$

where

$$
M_{ \pm}(\alpha)=2(1 \pm\langle\alpha \mid-\alpha\rangle)=2\left(1 \pm \exp \left(-2|\alpha|^{2}\right)\right.
$$

These states have a long history dating back to the original paper by Dodonov, Malkin and Man'ko [1]. and, apart from the fundamental interest, are shown to be technologically useful.

In metrology, even coherent states in combination with parity detection method are used to realize super-resolving angular rotation measurements [2]. Optical Schrödinger cat states can also serve as a basis for quantum computations [3], teleportation and cryptography [4-7]. These states have been produced experimentally using the optical Kerr effect in 1986 by Yurke and Stoler [8]. Among more recent experimental procedures suggested for generation of optical cat states are the method based on photon subtraction of an optical squeezed state [9-12] and the protocol that uses homodyne detection and photon number states [13] (see [14] for a review on generating cat states).

In this paper, our goal is to assess the fragility of the cat states propagating in noisy quantum channels. More specifically, we consider the statistics of photocounts underlying the method to discriminate between the parity of the cat states and examine its sensitivity to environment induced decoherence effects. We shall also calculate the fidelity of the states depending on the amplitude and the transmission of the fiber channel. In addition to the model of the quantum channel based on the beam splitter-like transformation acting in the enlarged Hilbert space, we employ Lindblad dynamics of an one-mode bosonic system to model a lossy optical fiber. 


\section{Results}

Following $[6,7,15]$, we begin with the simplest model of the noisy channel representing the fiber. In this model, the lossy mode is combined with the beam splitter of the transmission, $\tau$, along with the input vacuum state in additional environmental (ancillary) mode $E$ :

$$
\begin{aligned}
& \left|\alpha_{ \pm}\right\rangle \rightarrow\left|\Psi_{ \pm}\right\rangle=\hat{T}_{B S}(\tau)\left|\alpha_{ \pm}\right\rangle \otimes|0\rangle_{E} \\
& =\frac{1}{\sqrt{M_{ \pm}(\alpha)}}\left\{|\sqrt{\tau} \alpha\rangle \otimes|\sqrt{1-\tau} \alpha\rangle_{E} \pm|-\sqrt{\tau} \alpha\rangle \otimes|-\sqrt{1-\tau} \alpha\rangle_{E}\right\},
\end{aligned}
$$

where $\hat{T}_{B S}(\tau)$ is the unitary describing the beam splitter with the transmittance $\tau$. Clearly, $\left|\Psi_{ \pm}\right\rangle$is the entangled two-mode state generated by the beam splitter. Note that, according to Ref. [16], the phase modulator can be regarded as a multiport beam splitter that transforms a one-mode Schrödinger cat state into entangled multimode state.

The density operators can now be readily derived by tracing out the ancillary mode. The result:

$$
\hat{\rho}_{ \pm}=\operatorname{Tr}_{E}\left|\Psi_{ \pm}\right\rangle\left\langle\Psi_{ \pm}\left|=P_{ \pm}\right| \gamma_{ \pm}\right\rangle\left\langle\gamma_{ \pm}\left|+Q_{ \pm}\right| \gamma_{\mp}\right\rangle\left\langle\gamma_{\mp}\right|,
$$

where $\gamma \equiv \sqrt{\tau} \alpha$, shows that the density matrices are mixtures of even and odd cat states with the coefficients given by:

$$
\begin{aligned}
& P_{+}=\cosh \left((1-\tau)|\alpha|^{2}\right) \frac{\cosh \left(\tau|\alpha|^{2}\right)}{\cosh \left(|\alpha|^{2}\right)}, \quad Q_{+}=1-P_{+}=\sinh \left((1-\tau)|\alpha|^{2}\right) \frac{\sinh \left(\tau|\alpha|^{2}\right)}{\cosh \left(|\alpha|^{2}\right)} \\
& P_{-}=\cosh \left((1-\tau)|\alpha|^{2}\right) \frac{\sinh \left(\tau|\alpha|^{2}\right)}{\sinh \left(|\alpha|^{2}\right)}, \quad Q_{-}=1-P_{-}=\sinh \left((1-\tau)|\alpha|^{2}\right) \frac{\cosh \left(\tau|\alpha|^{2}\right)}{\sinh \left(|\alpha|^{2}\right)}
\end{aligned}
$$

Since $\left\langle\gamma_{\mu} \mid \gamma_{\nu}\right\rangle=\delta_{\mu \nu}$, the above coefficients $P_{\nu}$ and $Q_{\nu}$ are the eigenvalues of the density operator $\hat{\rho}_{\nu}$.

The effect of decoherence for even and odd cat states is characterized by the probabilities $Q_{+}$and $Q_{-}$. It is not difficult to see that, $Q_{ \pm}$tends to $1 / 2$ as $|\alpha|$ becomes infinitely large, and thus the operators $\hat{\rho}_{+}$and $\hat{\rho}_{-}$are both approaching the limit of the completely chaotic (equiprobable) mixture as $|\alpha|$ increases. For small $|\alpha|$ with $\alpha \approx 0, \hat{\rho}_{+}$ and $\hat{\rho}_{-}$are close to the vacuum state $|0\rangle\langle 0|$ and the mixed state $(1-\tau)|0\rangle\langle 0|+\tau| 1\rangle\langle 1|$, respectively.

Given the density operator (4), we can now evaluate the fidelity:

$$
F_{ \pm}=\left\langle\alpha_{ \pm}\left|\hat{\rho}_{ \pm}\right| \alpha_{ \pm}\right\rangle
$$

characterizing the difference between the mixed and pure states: $\hat{\rho}_{ \pm}$and $\left|\alpha_{ \pm}\right\rangle\left\langle\alpha_{ \pm}\right|$. For even and odd cat states, we have:

$$
\begin{aligned}
& F_{+}=\frac{\cosh \left((1-\tau)|\alpha|^{2}\right)}{\cosh ^{2}\left(|\alpha|^{2}\right)} \cosh ^{2}\left(\sqrt{\tau}|\alpha|^{2}\right), \\
& F_{-}=\frac{\cosh \left((1-\tau)|\alpha|^{2}\right)}{\sinh ^{2}\left(|\alpha|^{2}\right)} \sinh ^{2}\left(\sqrt{\tau}|\alpha|^{2}\right) .
\end{aligned}
$$

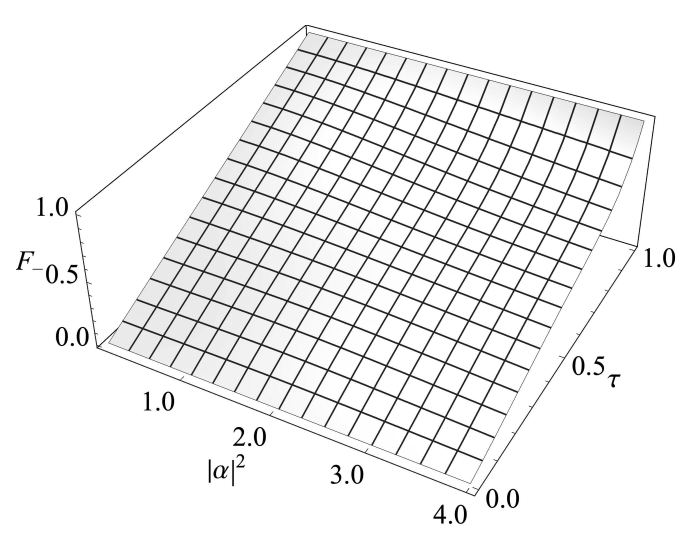

(A)

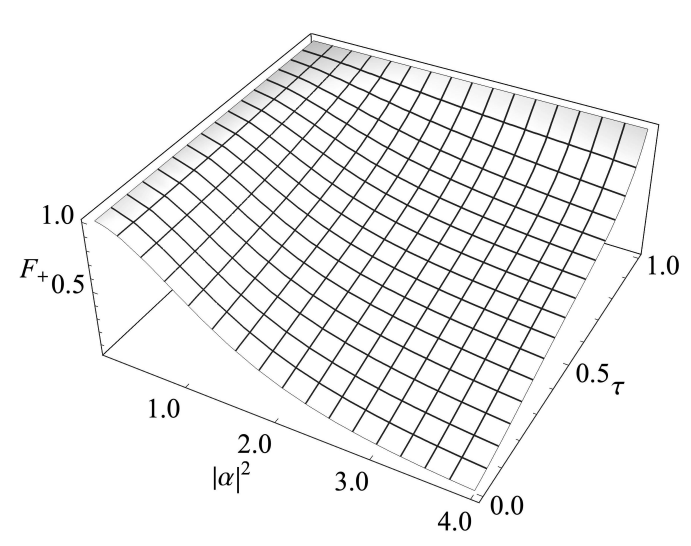

(B)

FIG. 1. Fidelity as a function of the transmittance coefficient $\tau$ and the mean photon number $|\alpha|^{2}$ for $(\mathrm{A})$ odd and $(\mathrm{B})$ even cat states 
Fig. 1 shows the fidelities $F_{-}$and $F_{+}$as an function of the transmittance $\tau$ the mean photon number $|\alpha|^{2}$. It is seen that, in the zero amplitude limit where the symmetric cat state is close the vacuum state, the fidelity $F_{+}$approaches unity. The corresponding value for the fidelity of the antisymmetric cats equals $\tau$. At large $|\alpha|$, the fidelitites are exponentially decaying functions of the mean photon number: $F_{ \pm} \approx \exp \left(-(1-\sqrt{\tau})^{2}|\alpha|^{2}\right)$.

Our next step is to study how decoherence that has a detrimental effect on the fidelity will affect the distinguishability of the cat states. For this purpose, we shall discuss performing photocounting measurements as the means to discriminate between the even and odd cat states based on the parity of the photon number registered by a photonnumber resolving photodetector.

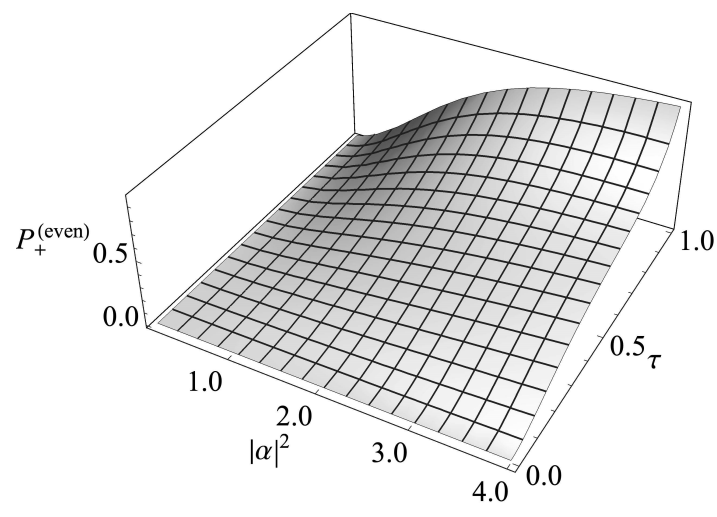

(A)

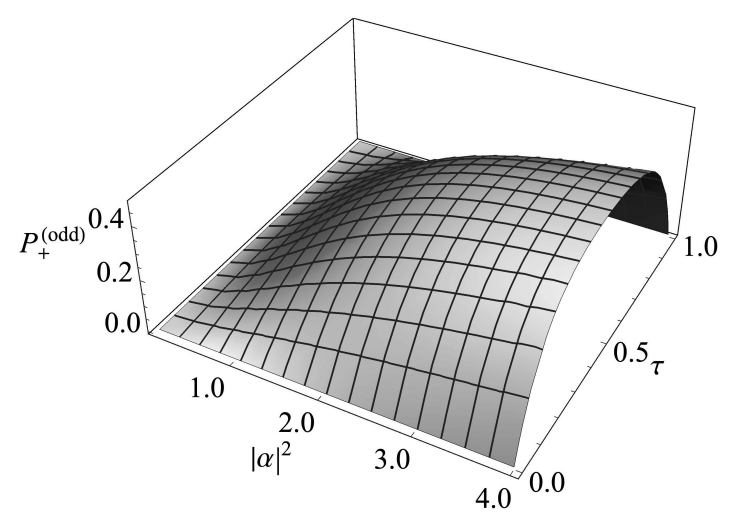

(B)

FIG. 2. Probabilities to detect (A) even and (B) odd number of photocounts as a function of the transmittance coefficient $\tau$ and the mean photon number $|\alpha|^{2}$ for the symmetric (even) Schrödinger cat

In order to evaluate the statistics of photocounts for the quantum cat states, $\hat{\rho}_{+}$and $\hat{\rho}_{-}$, we shall use the wellknown Kelley-Kleiner formula [17] and deduce the following expression for the probability to detect $k$ photons:

$$
\operatorname{Pr}_{\nu}(k)=\frac{1}{k !} \operatorname{Tr}\left[:\left(\eta_{\mathrm{d}} \hat{a}^{\dagger} \hat{a}\right)^{k} e^{-\eta_{\mathrm{d}} \hat{a}^{\dagger} \hat{a}}: \rho_{\nu}\right]=P_{\nu} C_{\nu}(k)+Q_{\nu} C_{-\nu}(k),
$$

where

$$
\begin{aligned}
& C_{\nu}(k) \equiv \frac{1}{k !}\left\langle\gamma_{\nu}\left|:\left(\eta_{\mathrm{d}} \hat{a}^{\dagger} \hat{a}\right)^{k} e^{-\eta_{\mathrm{d}} \hat{a}^{\dagger} \hat{a}}:\right| \gamma_{\nu}\right\rangle \\
& =\frac{\left(\eta_{\mathrm{d}} \tau|\alpha|^{2}\right)^{k}}{k !} \frac{\exp \left(\left(1-\eta_{\mathrm{d}}\right) \tau|\alpha|^{2}\right)+(-1)^{k} \nu \exp \left(-\left(1-\eta_{\mathrm{d}}\right) \tau|\alpha|^{2}\right)}{\exp \left(\tau|\alpha|^{2}\right)+\nu \exp \left(-\tau|\alpha|^{2}\right)},
\end{aligned}
$$

$\eta_{\mathrm{d}}$ is the efficiency of the photodetector.

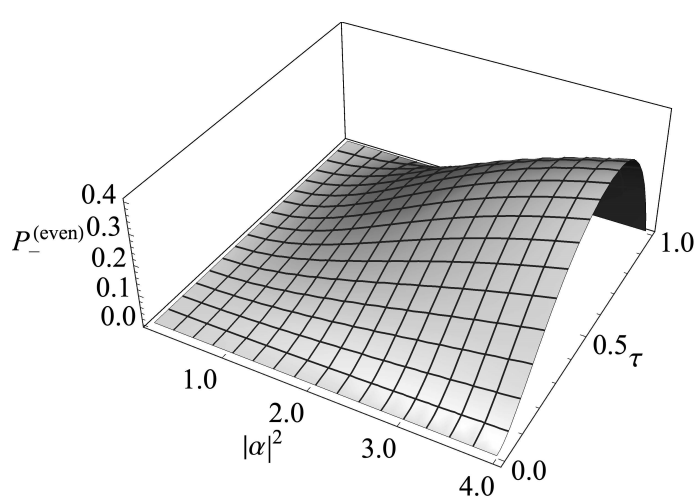

(A)

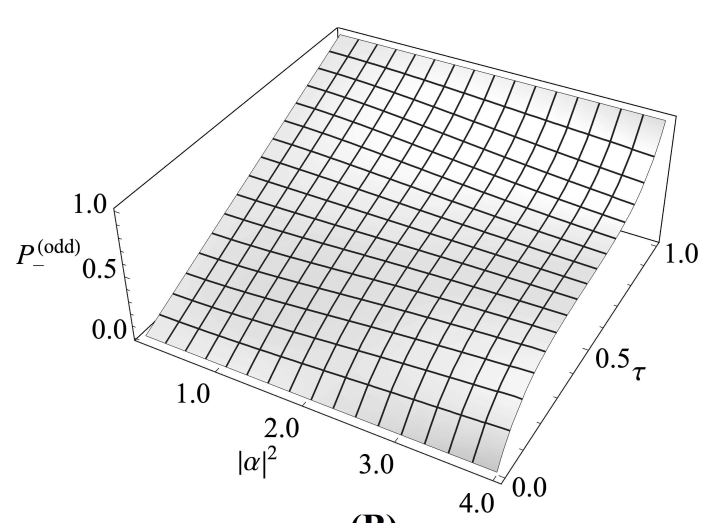

(B)

FIG. 3. Probabilities to detect (A) even and (B) odd number of photocounts as a function of the transmittance coefficient $\tau$ and the mean photon number $|\alpha|^{2}$ for the antisymmetric (odd) Schrödinger cat 
From Eq. (9) it can be readily seen that, in the case where the efficiency and the transmittance are both equal to unity, $\eta_{\mathrm{d}}=\tau=1$, the probability of odd (even) number of clicks:

$$
P_{\nu}^{(\text {odd })}=\sum_{k=0}^{\infty} \operatorname{Pr}_{\nu}(2 k+1), \quad P_{\nu}^{(\text {even })}=\sum_{k=1}^{\infty} \operatorname{Pr}_{\nu}(2 k)
$$

vanishes for the even (odd) states, $P_{+}^{(\text {odd })}=0\left(P_{-}^{(\text {even })}=0\right)$, and thus the cat states are perfectly distinguishable. Note that, in contrast to the case of pure odd cat state, where the no-click probabilty is zero, $P_{-}(0)=0$, for the pure even cat state, this probability decays with $|\alpha|^{2}, P_{+}(0)=1-P_{+}^{(\text {even })}=1 / \cosh \left(|\alpha|^{2}\right)$, and represents the non-vanishing contribution of the vacuum state.

As is shown in Figs. 2 and 3, dependence of the decoherence induced probabilities $P_{+}^{(\text {odd) }}$ and $P_{-}^{(\text {even })}$ on the channel transmittance reveal nonmonotonic behavior. From the above discussion, these probabilities equal zero in the limit of the lossless channel with $\tau=1$. In the opposite case, with $\tau=0$, the density operators are projectors on the vacuum state $|0\rangle$ and $P_{ \pm}^{(\text {odd })}=P_{ \pm}^{(\text {even })}=0$. So, referring to Fig. 4 , the no-click probabilities $P_{+}(0)$ and $P_{-}(0)$ are both equal to unity in the no-signal limit with $\tau=0$.

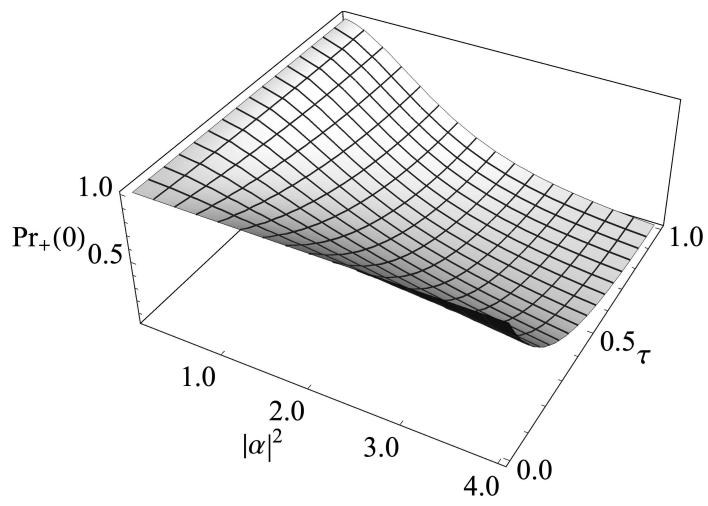

(A)

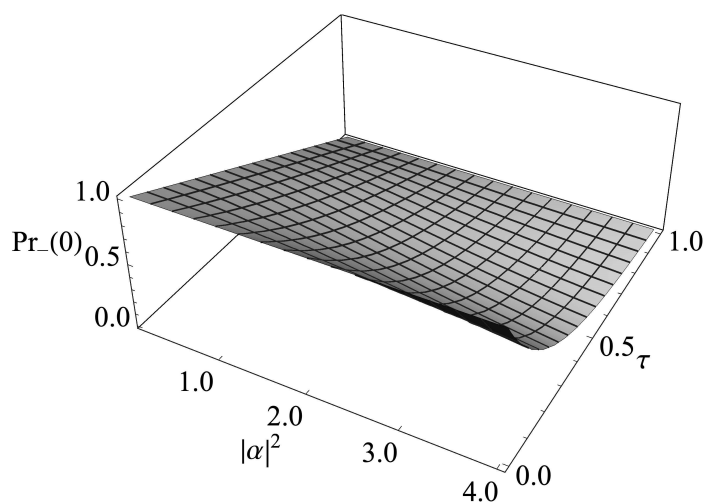

(B)

FIG. 4. No-click probability as a function of the transmittance coefficient $\tau$ and the mean photon number $|\alpha|^{2}$ for (A) symmetric (even) and (B) antisymmetric (odd) Schrödinger cats

The results indicate that the statistics of photocounts for the odd cat state at small $|\alpha|^{2}$ are less sensitive to the decoherence effects as compared to the even states. Interestingly, there is the optimal value of $|\alpha|$ that gives the greatest possible difference $P_{+}^{(\text {even })}-P_{+}^{(\text {odd })}$, whereas $P_{-}^{\text {(odd) }}-P_{-}^{(\text {even) }}$ monotonically decreases with $|\alpha|$.

An alternative approach to modelling of the quantum channel representing a noisy fiber is based on the master equation method $[16,18,19]$. In our case we can start from the one-mode Markovian thermal bath version of the Lindblad equation for the density matrix given by:

$$
\begin{aligned}
& \frac{\partial \hat{\rho}}{\partial t}=\mathcal{L} \hat{\rho}=-i \Omega[\hat{n}, \hat{\rho}]+\kappa\left(\mathcal{D}_{\hat{a} \hat{a}^{\dagger}} \hat{\rho}+\mathrm{e}^{-z_{T}} \mathcal{D}_{\hat{a}^{\dagger} \hat{a}} \hat{\rho}\right), \\
& \hat{n}=\hat{a}^{\dagger} \hat{a}, \quad \mathcal{D}_{\hat{A} \hat{B}} \hat{\rho} \equiv 2 \hat{A} \hat{\rho} \hat{B}-\hat{B} \hat{A} \hat{\rho}-\hat{\rho} \hat{B} \hat{A},
\end{aligned}
$$

where the dagger denotes Hermitian conjugation, $\hat{\rho}$ is the density matrix representing the quantum state; $\hat{a}^{\dagger}(\hat{a})$ is the creation (annihilation) operator; $[\hat{A}, \hat{B}]=\hat{A} \hat{B}-\hat{B} \hat{A}$ stands for the commutator; $\Omega(\kappa)$ is the element of the frequency (relaxation) constant, $z_{T}=\frac{\hbar \Omega_{0}}{k_{B} T}$ is the dimensionless inverse temperature parameter, where $\Omega_{0}$ is the bare frequency, $\hbar$ is the reduced Planck constant, $k_{B}$ is the Boltzmann constant and $T$ is the temperature of the environment.

By using the method of characteristics, it can be shown that temporal evolution of the normally ordered characteristic function is described by the relation [20]:

$$
\chi_{N}(\beta, t)=\operatorname{Tr}\left[\mathrm{e}^{\beta \hat{a}^{\dagger}} \mathrm{e}^{-\beta^{*} \hat{a}} \hat{\rho}(t)\right]=\mathrm{e}^{-\langle n\rangle|\beta|^{2}} \chi_{\mathrm{ini}}\left(\mathrm{e}^{(i \Omega-\Gamma) t} \beta\right), \quad\langle n\rangle=\left(1-\mathrm{e}^{-2 \Gamma t}\right) n_{T}
$$


where $\Gamma=\left(1-\mathrm{e}^{-z_{T}}\right) \kappa$ is the relaxation rate; $n_{T}=\left(\mathrm{e}^{z_{T}}-1\right)^{-1}$ is the mean number of thermal photons; and $\chi_{\mathrm{ini}}(\beta)$ is determined by the initial condition $\hat{\rho}(0)=\left|\alpha_{\nu}\right\rangle\left\langle\alpha_{\nu}\right|$ as follows:

$$
\begin{aligned}
& \chi_{\mathrm{ini}}(\beta)=\chi_{\nu}(\beta)=\left\langle\alpha_{\nu}\left|\mathrm{e}^{\beta \hat{a}^{\dagger}} \mathrm{e}^{-\beta^{*} \hat{a}}\right| \alpha_{\nu}\right\rangle \\
& =\frac{\cosh \left(\beta \alpha^{*}-\beta^{*} \alpha\right)+\nu \mathrm{e}^{-2|\alpha|^{2}} \cosh \left(\beta \alpha^{*}+\beta^{*} \alpha\right)}{1+\nu \mathrm{e}^{-2|\alpha|^{2}}} .
\end{aligned}
$$

An important point is that the probability of clicks (8) is expressed in terms of the moments $\left\langle: \hat{n}^{m}:\right\rangle$ :

$$
\operatorname{Pr}_{\nu}(k)=\sum_{m=0}^{\infty} \frac{(-1)^{m} \eta_{\mathrm{d}}^{m+k}}{k ! m !}\left\langle: \hat{n}^{m+k}:\right\rangle
$$

In particular, for the cat states, generating function of the moments:

$$
\begin{aligned}
& \tilde{\chi}_{\nu}(|\beta|)=\frac{1}{2 \pi} \int_{0}^{2 \pi} \chi_{\nu}\left(|\beta| \mathrm{e}^{i \phi}\right) \mathrm{d} \phi=\sum_{m=0}^{\infty} \frac{(-1)^{m}|\beta|^{2 m}}{(m !)^{2}}\left\langle: \hat{n}^{m}:\right\rangle_{\nu}, \\
& \left\langle: \hat{n}^{m}:\right\rangle_{\nu}=\frac{1+(-1)^{m} \nu \mathrm{e}^{-2|\alpha|^{2}}}{1+\nu \mathrm{e}^{-2|\alpha|^{2}}}|\alpha|^{2 m}
\end{aligned}
$$

is obtained by averaging $\chi_{\nu}(\beta)$ over the phase of $\beta$ and leads to the expression for the probability of photocounts given by Eq. (9). Then the phase averaged characteristic function (12):

$$
\tilde{\chi}_{N}(|\beta|, t)=\frac{1}{2 \pi} \int_{0}^{2 \pi} \chi_{N}\left(|\beta| \mathrm{e}^{i \phi}, t\right) \mathrm{d} \phi=\mathrm{e}^{-\langle n\rangle|\beta|^{2}} \tilde{\chi}_{\nu}(\sqrt{T}|\beta|)=\sum_{m=0}^{\infty} \frac{(-1)^{m}|\beta|^{2 m}}{(m !)^{2}}\left\langle: \hat{n}^{m}:\right\rangle
$$

gives the expression for the normally ordered moments of the photon number operator

$$
\left\langle: \hat{n}^{m}:\right\rangle=\sum_{l=0}^{m}\left[\frac{m !}{(m-l) !}\right]^{2} \frac{\langle n\rangle^{l}}{l !} T^{m-l}\left\langle: \hat{n}^{m-l}:\right\rangle_{\nu}
$$

where $T=\mathrm{e}^{-2 \Gamma t}$ is the channel transmittance. Formula (17) can now be combined with Eq. (14) to compute the probability of photocounts (10). It is not difficult to see that, at $\eta_{\mathrm{d}}=1$, the probabilities $P_{+}^{\text {(odd) }}$ and $P_{-}^{\text {(even) }}$ vanish in the zero-temperature limit with $n_{T}=0$. Thus we arrive at the conclusion that within the framework of Lindblad dynamics distinguishability of the Schrödinger cat states is solely governed by the mean number of thermal photons $\langle n\rangle \propto n_{T}$ rather than the channel transmittance.

\section{Conclusion and discussion}

We have studied the fidelity and the statistics of photocounts for the even and odd Shrödinger cat states subjected to the effects of decoherence in the noisy quantum channel representing a lossy optical fiber. For the beam splitter model of the quantum channel characterized by the transmittance $\tau$, the probabilities that determine the photocounting statistics are computed in realtion to $\tau$ and the mean photon number $|\alpha|^{2}$. By contrast to this model, exact results describing Lindblad dynamics of photon states show that the key parameter governing decoherence induced effects on photocounting is the mean number of thermal photons $n_{T}$.

In our analysis, the criterion used to discriminate between the even and odd states is based on the parity of photocounts registered by a photodetector. Since the vacuum state contribution to the even Schrödinger cat states is found to reduce their distinguishability, the odd coherent states present the most robust case.

In general, from our results, it might be concluded that, despite daunting nature of the task to utilize Schrödinger cat states for quantum communications, these states can be used in entanglement distribution (swapping) protocols at sufficiently short distances.

\section{Acknowledgements}

The work was done by Leading Research Center "National Center of Quantum Internet" of ITMO University during the implementation of the government support program, with the financial support of Ministry of Digital Development, Communications and Mass Media of the Russian Federation and RVC JSC; Grant Agreement ID: 0000000007119P190002, agreement No. 006-20 dated 27.03.2020. A.D.K. acknowledges financial support by the Ministry of Education and Science of the Russian Federation (Grant No. 2019-0903). 


\section{References}

[1] Dodonov V.V., Malkin I.A., Man'ko V.I. Even and odd coherent states and excitations of a singular oscillator, Physica, 1974, 72(3), P. 597-615.

[2] Hao L., Tang H., Wang Q. Resolution improvement of angular rotation measurement through even coherent states coupled with parity detection strategy. Journal of Optics, 2020, 22(2), P. 025203.

[3] Lund A.P., Ralph T.C., Haselgrove H.L. Fault-tolerant linear optical quantum computing with small-amplitude coherent states. Phys. Rev. Lett., 2008, 100, P. 030503.

[4] Sangouard N., Simon C., Gisin N., Laurat J., Tualle-Brouri R., Grangier P. Quantum repeaters with entangled coherent states. J. Opt. Soc. Am. B, 2010, 27(6), P. A137-A145.

[5] Brask J.B., Rigas I., Polzik E.S., Andersen U.L., Sørensen A.S. Hybrid long-distance entanglement distribution protocol. Phys. Rev. Lett., 2010, 105, P. 160501.

[6] Ghasemi M., Tavassoly M.K. Toward a quantum repeater protocol based on the coherent state approach. Laser Physics, $2019,29$.

[7] Parker R.C., Joo J., Spiller T.P. Photonic hybrid state entanglement swapping using cat state superpositions. Proceedings of the Royal Society A: Mathematical, Physical and Engineering Sciences, 2020, 476(2243), P. 20200237.

[8] Yurke B., Stoler D. Generating quantum mechanical superpositions of macroscopically distinguishable states via amplitude dispersion. Phys. Rev. Lett., 1986, 57, P. 13-16.

[9] Neergaard-Nielsen J.S., Nielsen B.M., Hettich C., Mølmer K., Polzik E.S., Generation of a superposition of odd photon number states for quantum information networks. Phys. Rev. Lett., 2006, 97, P. 083604.

[10] Ourjoumtsev A., Ferreyrol F., Tualle-Brouri R., Grangier P. Preparation of non-local superpositions of quasi-classical light states. Nature Physics, 2009, 5, P. 189-192.

[11] Serikawa T., Yoshikawa J.-i., Takeda S., Yonezawa H., Ralph T.C., Huntington E.H., Furusawa A. Generation of a cat state in an optical sideband. Phys. Rev. Lett., 2018, 121, P. 143602.

[12] Takase K., Yoshikawa J.-i., Asavanant W., Endo M., Furusawa A. Generation of optical Schrödinger cat states by generalized photon subtraction. Phys. Rev. A, 2021, 103, P. 013710.

[13] Ourjoumtsev A., Jeong H., Tualle-Brouri R., Grangier P. Generation of optical "Schrödinger cats" from photon number states. Nature, 2007, 448, P. 784-786.

[14] Glancy S., de Vasconcelos H.M. Methods for producing optical coherent state superpositions. J. Opt. Soc. Am. B, 2008, 25(5), P. 712-733.

[15] S.J. van Enk, Hirota O. Entangled coherent states: Teleportation and decoherence. Phys. Rev. A, 2001, 64, P. 022313.

[16] Miroshnichenko G.P., Kiselev A.D., Trifanov A.I., Gleim A.V. Algebraic approach to electro-optic modulation of light: exactly solvable multimode quantum model. J. Opt. Soc. Am. B, 2017, 34(6), P. 1177-1190.

[17] Kelley P.L., Kleiner W.H. Theory of electromagnetic field measurement and photoelectron counting. Phys. Rev., 1964, 136, P. A316-A334.

[18] Kozubov A., Gaidash A., Miroshnichenko G. Quantum model of decoherence in the polarization domain for the fiber channel. Phys. Rev. A, 2019, 99, P. 053842.

[19] Gaidash A., Kozubov A., Miroshnichenko G. Dissipative dynamics of quantum states in the fiber channel. Phys. Rev. A, 2020 , 102, P. 023711.

[20] Kiselev A.D., Ali R., Rybin A.V. Lindblad dynamics and disentanglement in multi-mode bosonic systems. Entropy, 2021, 23(11). 